\title{
Manejo de la embarazada con isoinmunización por anticuerpos irregulares
}

\author{
Javiera Fuenzalida C. ${ }^{1}$, Jorge A. Carvajal C. ${ }^{1,2}$ \\ ${ }^{1}$ División de Obstetricia y Ginecología, ${ }^{2}$ Unidad de Medicina Materno-Fetal, Facultad de Medicina, Escuela de Medicina, \\ Pontificia Universidad Católica de Chile.
}

\section{RESUMEN}

La isoinmunización eritrocitaria feto-materna se define como la presencia de anticuerpos maternos dirigidos contra antígenos presentes en los glóbulos rojos fetales. Los anticuerpos maternos pueden atravesar la barrera placentaria y provocar hemólisis de los glóbulos rojos fetales produciendo anemia hemolítica e hiperbilirrubinemia, características de la enfermedad hemolítica perinatal (EHP). La principal causa de EHP es la incompatibilidad $A B O$, seguida de la isoinmunización por $\mathrm{RhD}$; esta última ha disminuido su incidencia dado el amplio uso de inmunoglobulina anti D. Sin embargo, el glóbulo rojo tiene más de 400 antígenos, muchos de ellos (>50) capaces de producir isoinmunización y EHP. En este artículo, revisamos la evidencia y proponemos un algoritmo de manejo y seguimiento de las embarazadas con isoinmunización por anticuerpos irregulares. En la isoinmunización por anticuerpos irregulares, los títulos de anticuerpos maternos no se correlacionan con la gravedad de la enfermedad. La anemia en la EHP por anticuerpos anti-Kell es secundaria a una supresión de la eritroblastosis fetal a diferencia del resto de los sistemas que producen anemia hemolítica. Recomendamos efectuar tamizaje de todas las pacientes en el control prenatal, solicitando grupo sanguíneo, Rh y test de Coombs indirecto. En las pacientes Rh (+) con test de Coombs indirecto positivo es necesario identificar los anticuerpos irregulares. En caso de tener isoinmunización por anticuerpos irregulares con riesgo de EHP, derivar a una unidad de alto riesgo obstétrico para realizar seguimiento de la aparición de anemia fetal midiendo de modo seriado el peak sistólico de la arteria cerebral media. Si se detecta anemia fetal, debemos planificar una cordocentesis para confirmar el diagnóstico y tratar la anemia.

\section{PALABRAS CLAVE: Isoinmunización, anticuerpos irregulares, enfermedad hemolítica perinatal}

\section{SUMMARY}

The fetomaternal erythrocyte isoimmunization is defined as the presence of maternal antibodies directed against antigens present in fetal red blood cells. Maternal antibodies can cross the placenta and cause hemolysis of fetal red blood cells causing hyperbilirubinemia and hemolytic anemia, known as perinatal hemolytic disease (PHD). The main cause of PHD is ABO incompatibility, followed by RhD isoimmunization. The latter has decreased its incidence since the widespread use of anti-D immunoglobulin. However, the red cell has more than 400 antigens; many of them $(>50)$ can lead to isoimmunization and PHD. In this article, we review the evidence and propose an algorithm for the management and monitoring of pregnant women with irregular antibodies isoimmunization. In the isoimmunization by irregular antibodies, maternal antibody titers do not correlate with the severity of the disease. Anemia in PHD by anti-Kell is secondary to suppression of fetal erythroblastosis unlike other systems that produce hemolytic anemia. We suggest the screening of all patients in the prenatal control with blood group, Rh and indirect Coombs test. In the Rh (+) patients with 
positive indirect Coombs test, irregular antibodies should be identified. In case of immunization by irregular antibodies in risk of PHD, the women should be referred to a high-risk obstetrics where the appearance of fetal anemia should be seek by measuring systolic peak of middle cerebral artery. If anemia is detected full confirmation of diagnosis and treatment should be effected by cordocentesis.

\section{KEY WORDS: Isoimmunization, irregular antibodies, perinatal hemolytic disease}

\section{INTRODUCCIÓN}

La incompatibilidad eritrocitaria se define como la presencia de uno o más antígenos en el glóbulo rojo fetal que no están presentes en el glóbulo rojo materno. Esta incompatibilidad eritrocitaria feto-materna puede generar una respuesta inmune materna mediada por inmunoglobulinas, desencadenando lo que se denomina isoinmunización eritrocitaria feto materna. La isoinmunización eritrocitaria feto materna, también llamada aloinmunización, se define como la presencia de anticuerpos maternos dirigidos contra antígenos presentes en los glóbulos rojos fetales (1).

Los anticuerpos maternos resultan de la respuesta inmune a un contacto anterior con los antígenos durante una transfusión sanguínea, un embarazo previo, el mismo embarazo o un trasplante. Los anticuerpos maternos pueden atravesar la barrera placentaria y provocar hemólisis de los glóbulos rojos fetales portadores del antígeno. La hemólisis de los glóbulos rojos fetales provoca anemia hemolítica e hiperbilirrubinemia características de la enfermedad hemolítica perinatal (EHP) o eritroblastosis fetal $(1,2)$.

La EHP tiene un amplio espectro de presentación desde un cuadro subclínico hasta hidrops fetal y muerte intrauterina. Se clasifica de leve a severa según el grado de anemia y niveles de bilirrubina. La EHP leve es aquella con valores de hemoglobina mayores a $12 \mathrm{~g} / \mathrm{dl}$, con bilirrubina que no excede $16-20 \mathrm{~g} / \mathrm{dl}$ y que en general no requiere tratamiento postnatal o solo fototerapia. La EHP moderada se presenta con valores de hemoglobina generalmente mayores a $9 \mathrm{~g} / \mathrm{dl}$, con anemia moderada e ictericia que puede requerir transfusión o exanguíneo-transfusión. Finalmente, la EHP severa es aquella que se presenta con anemia severa, con hematocrito menor a $15 \%$ e hidrops fetal que requiere transfusión intrauterina o interrupción del embarazo dependiendo de la edad gestacional (3).

La principal causa de EHP es la incompatibilidad $A B O$, seguida de la isoinmunización por $\mathrm{RhD}$. El amplio uso de inmunoglobulina anti $D$ para la prevención de la isoinmunización por $\mathrm{RhD}$ en mujeres RhD (-) no sensibilizadas ha disminuido la incidencia de EHP secundaria a isoinmunización por $\mathrm{RhD}$. Sin embargo, el glóbulo rojo tiene más de 400 antígenos, de los cuales se han descrito más de 50 relacionados con el desarrollo de EHP en todo su espectro (Tabla I) $(4,5)$.

Los antígenos de los glóbulos rojos son parte de glicoproteínas expuestas en la superficie extracelular de la membrana del eritrocito. En la nomenclatura de los grupos sanguíneos, los antígenos codificados por el mismo gen o grupo de genes se asignan al mismo sistema, de esta forma, cada sistema consiste en uno o más antígenos. Los más comúnmente reconocidos son los del grupo $A B O$ y los del sistema Rhesus ( $D, d, E, e, C, c)$. Además, existe un grupo de otras glicoproteínas menos frecuentes, que también pueden estar presentes en la membrana de los eritrocitos como el sistema Kell, Duffy, MNSS, Lewis y Kidd, todos capaces de generar una respuesta inmune. En general, los anticuerpos anti glóbulos rojos distintos al $\mathrm{RhD}$, son llamados anticuerpos irregulares o anticuerpos no clásicos y pueden ser detectados en la sangre materna mediante el test de Coombs indirecto (6).

La prevalencia de anticuerpos maternos contra antígenos fetales varía en las distintas poblaciones. Los estudios muestran un $1,2 \%$ de mujeres embarazadas aloinmunizadas, un tercio de los casos corresponde a anticuerpos contra antígenos capaces de producir enfermedad hemolítica perinatal, principalmente RhD, Kell, RhE y Rhc $(7,8)$.

Los factores de riesgo más importantes para presentar isoinmunización eritrocitaria son la multiparidad o la exposición a transfusiones sanguíneas. Existe un estudio retrospectivo que analiza los antecedentes de las mujeres embarazadas con diagnóstico de isoinmunización eritrocitaria no $\mathrm{RhD}$. Sus autores concluyen que el factor de riesgo más importante es el antecedente de transfusión sanguínea, principalmente para las pacientes con anticuerpos anti-Kell, de las cuales un $83 \%$ había recibido una transfusión, seguido de la paridad, antecedente de cirugía mayor o enfermedad hematológica (9). 
Tabla I

ANTÍGENOS DEL GLÓBULO ROJO FETAL CAPACES DE PRODUCIR ISOINMUNIZACIÓN FETO MATERNA

\begin{tabular}{|c|c|c|}
\hline Sistema & Antígenos & EHP \\
\hline \multicolumn{3}{|c|}{ Más comunes } \\
\hline Lewis & & No (IgM) \\
\hline I & & No (IgM) \\
\hline \multirow[t]{7}{*}{ Kell } & Kell & Leve a severa \\
\hline & Cellano & Leve \\
\hline & Ko & Leve \\
\hline & $K p^{a}$ & Leve \\
\hline & $\mathrm{Kp} \mathrm{p}^{\mathrm{b}}$ & Leve \\
\hline & $\mathrm{Js}^{\mathrm{a}}$ & Leve \\
\hline & $\mathrm{Js}^{\mathrm{b}}$ & Leve \\
\hline \multirow[t]{4}{*}{$\mathrm{Rh}$} & E & Leve a severa \\
\hline & e & Leve a severa \\
\hline & C & Leve a severa \\
\hline & c & Leve a severa \\
\hline \multirow[t]{3}{*}{ Duffy } & $\mathrm{Fy}^{\mathrm{a}}$ & Leve a severa \\
\hline & $\mathrm{Fy}^{\mathrm{b}}$ & No \\
\hline & $\mathrm{By}^{3}$ & Leve \\
\hline \multirow[t]{3}{*}{ Kidd } & $\mathrm{Jk}^{\mathrm{a}}$ & Leve a severa \\
\hline & $\mathrm{Jk}^{\mathrm{b}}$ & Leve \\
\hline & $\mathrm{Jk}^{3}$ & Leve \\
\hline \multirow[t]{5}{*}{ MNSs } & M & Leve a severa \\
\hline & $\mathrm{N}$ & Leve \\
\hline & S & Leve a severa \\
\hline & s & Leve a severa \\
\hline & $U$ & Leve a severa \\
\hline
\end{tabular}

Otros grupos

\begin{tabular}{lll} 
MSSs & Mta & Moderado \\
& Vw & Leve \\
& Mur & Leve \\
& Hil & Leve \\
& Hut & Leve \\
Lutheran & Lua & Leve \\
Diego & Lub & Leve \\
& Dla & Leve a severa \\
& Dib & Leve a severa \\
\hline
\end{tabular}

Adaptado de ACOG Practice Bulletin (5). Se excluye de esta tabla a los dos principales causantes de la enfermedad hemolítica perinatal (EHP): ABO y RhD.
De esto se desprende la importancia de las transfusiones con pruebas cruzadas adecuadas que incluyan todos los anticuerpos eritrocitarios capaces de provocar enfermedad hemolítica, principalmente en mujeres en edad fértil.

En relación al manejo de la aloinmunización por anticuerpos irregulares en la embarazada, la evidencia disponible consiste en series de casos y experiencias de distintos centros que, en general, utilizan un manejo similar al de la isoinmunización por anticuerpos anti-D. Aquí revisamos las publicaciones acerca del manejo de las pacientes isoinmunizadas por grupos no $\mathrm{RhD}$ y proponemos un esquema de manejo basado en la evidencia disponible.

\section{ISOINMUNIZACIÓN POR ANTICUERPOS IRREGULARES}

\section{Sistema Rhesus (no RhD)}

El sistema Rhesus está compuesto por tres pares de antígenos: Cc, Dd y Ee según la nomenclatura de Fisher y Race. La presencia del antígeno $D$ determina el grupo "RhD positivo" y la ausencia del antígeno D determina el grupo "RhD negativo" (10). A continuación nos referiremos a los antígenos de este grupo, excluyendo el D.

Rhc: Es un antígeno de la familia Rhesus asociado a enfermedad hemolítica perinatal severa, el efecto hemolítico es similar al del RhD (6). Como antecedente importante destaca la presencia de transfusiones previas en un 30 a $50 \%$ de las pacientes aloinmunizadas (11). Existen diversas series de casos clínicos publicados en que se reporta enfermedad leve a severa (12-15). La serie más reciente reportó un $26 \%$ de enfermedad hemolítica perinatal severa con un $67 \%$ de transfusiones neonatales en madres isoinmunizadas (15). En cuanto a los títulos de anticuerpos relacionados con la presencia de enfermedad hemolítica, la mayoría de los casos más severos ocurrieron con títulos mayores o iguales a 1:16, sin embargo existieron casos con títulos menores (12-15).

RhC, RhE y Rhe. Existe poca información publicada acerca de la isoinmunización por estos antígenos. Generalmente, están presentes en títulos bajos en conjunto con anticuerpos anti-RhD y aparentemente, su presencia puede ser aditiva al efecto de este anticuerpo. Las transfusiones intrauterinas son infrecuentes en enfermedad hemolítica perinatal por estos anticuerpos (4). En las series de casos publicados por isoinmunización por RhC, la mayoría de los recién nacidos no fueron afectados por enfermedad hemolítica perinatal; sin embargo, un grupo de ellos requirió tratamiento posparto por enfermedad moderada a severa $(16,17)$. En relación a la isoinmunización por $\mathrm{RhE}$, 
la mayoría de los recién nacidos afectados por enfermedad hemolítica perinatal tuvieron un cuadro leve, sin embargo un pequeño grupo requirió tratamiento post parto con fototerapia y exanguineo transfusión. Los títulos de anticuerpos no se correlacionaron con la magnitud de la eritoblastosis fetal (18).

\section{Sistema Kell}

El sistema kell está compuesto por 24 antígenos que se designan por nombre propio, letra abreviada o número. Al menos 8 de estos antígenos se han relacionado con enfermedad hemolítica perinatal; los más comunes son Kell (abreviado como Ko K1) y Cellano (abreviado como k o K2) (4).

Kell (K; K1). El mecanismo de producción de anemia fetal en estos anticuerpos difiere del descrito para el resto de los grupos con riesgo de enfermedad hemolítica perinatal. El sistema Kell produce anemia por dos mecanismos: hemólisis de los glóbulos rojos y predominantemente por una inhibición de los precursores eritropoyéticos a nivel de la médula ósea fetal. Los estudios tanto in vivo como in vitro demuestran reticulocitosis y eritroblastosis disminuidas y niveles de bilirrubina que no se correlacionaban con el grado de anemia fetal (19-21). Existen diversas series de casos de pacientes con isoinmunización anti-kell que muestran resultados con enfermedad de leve a muy severa, incluyendo óbito fetal y muerte perinatal (22-27). En relación a los títulos de anticuerpos, debemos destacar que la presencia de EHP no se relaciona con los títulos de anticuerpos (24-27).

Cellano (k; K2). Existen pocos casos reportados en la literatura de enfermedad hemolítica perinatal secundaria a la presencia de este antígeno, con cuadros de leves a severos que requirieron exanguíneo transfusión (28-30). Los títulos de anticuerpos no se relacionan con la gravedad de la enfermedad. Los distintos autores sugieren que este antígeno se comportaría en forma similar a Kell produciendo supresión a nivel de la médula eritropoyética (4).

\section{Sistema MNS}

El sistema MNS está compuesto por 40 antígenos, entre ellos solamente $\mathrm{M}, \mathrm{N}, \mathrm{S}$, s y U se han asociado a enfermedad hemolítica perinatal (4).

M. El anticuerpos anti M es de tipo IgM. Se presenta como una aglutinina en frío. La conversión a IgG ocurre en raras ocasiones y se ha asociado a enfermedad hemolítica perinatal (4). Los anticuerpos anti-M están presentes en un $10 \%$ de los embarazos con tamizaje positivo, sin embargo rara vez se asocia con enfermedad hemolítica perinatal (31). Se han reportado pocos casos de enfermedad hemolítica perinatal secundaria a anticuerpos anti-M, la mayoría de los casos son leves requiriendo solo fototerapia, sin embargo hay casos reportados de enfermedad severa. Ninguna de las publicaciones relaciona los títulos de anticuerpos con la gravedad de la enfermedad $(32,33)$. Cabe destacar la importancia de solicitar la diferenciación del anticuerpo en IgM o IgG, dado que solo lgG puede atravesar la barrera placentaria y causar enfermedad fetal (4).

S: Las pacientes con anticuerpos anti-S tienen el antecedente de transfusiones sanguíneas en más de la mitad de los casos. En general produce enfermedad leve, sin embargo puede generar enfermedad severa $(4,34,35)$.

U: Es un antígeno poco común, presente principalmente en individuos de raza negra, pero es una causa reconocida de EHP de leve a severa $(4,36)$.

\section{Sistema Duffy}

El sistema Duffy está compuesto de dos antígenos, Fya y Fyb, solo el primero de los cuales se ha relacionado con enfermedad hemolítica perinatal (4). Duffy es un anticuerpo que pude ser responsable de accidentes hemolíticos, algunas veces letales, durante las transfusiones sanguíneas y es una causa ocasional de EHP (37). En una serie descrita, la EHP ocurrió en un $11 \%$ de los recién nacidos de madres con anti-Fya y fue de leve a moderada. No se reportó hidrops fetal o muerte atribuida a este cuadro (38).

\section{Sistema Kidd}

El sistema Kidd está compuesto por dos antígenos, el Jka y Jkb. En este grupo, se han descrito tanto reacciones transfusionales agudas y crónicas, como enfermedad hemolítica perinatal. En general, la enfermedad hemolítica perinatal es poco frecuente, leve y de buen pronóstico, sin embargo se han reportado casos severos incluso con resultado de pérdida fetal (39).

\section{ISOINMUNIZACIÓN POR MÚLTIPLES ANTICUERPOS}

Existe la inmunización materna por múltiples anticuerpos. En una serie de 24 embarazos con isoinmunización por múltiples anticuerpos, se identificaron 17 que tenían dos anticuerpos y 7 más de dos anticuerpos. Once pacientes (46\%) requirieron al menos una transfusión intrauterina. Los cuadros de enfermedad hemolítica perinatal más graves ocurrieron en las pacientes con anti-D y anti-C con o sin anti-E. La ausencia de anticuerpos anti-D se asoció con la menor necesidad de transfusiones intrauterinas. El total de transfusiones fue significativamente mayor comparado con un 
grupo de pacientes con solo anticuerpos anti-D. La presencia de anticuerpos anti-D, parece ser el factor más importante en el curso de una isoinmunización por múltiples anticuerpos, asimismo la presencia de otro anticuerpo, además del anti-D, aumentaría el riesgo de transfusión intrauterina (40).

\section{MANEJO}

El manejo de las pacientes con isoinmunización por grupos no Rh-D, se ha realizado clásicamente siguiendo las pautas utilizadas en el grupo $\mathrm{RhD}$, sin embargo existen ciertas diferencias entre el comportamiento de los distintos sistemas que hacen importante un manejo diferencial.

Estado antigénico fetal. En las pacientes con aloinmunización eritrocitaria sería importante el conocimiento de la presencia o no de estos antígenos en el feto para el manejo del embarazo, pues si el feto es negativo para el antígeno no hay riesgo perinatal.

En general, los antígenos del glóbulo rojo son sistemas bi-alélicos codominantes y si el padre es heterocigoto para cierto antígeno, el feto tiene un $50 \%$ de probabilidades de ser portador. Si el feto heredó el antígeno paterno y por lo tanto es portador, se debe realizar un seguimiento en unidades de alto riesgo obstétrico por el riesgo de desarrollar enfermedad hemolítica perinatal. Tradicionalmente, el estudio antigénico fetal se ha realizado mediante amniocentesis, un procedimiento invasivo no exento de riesgos que además podría provocar la sensibilización materna (41).

El análisis del ADN fetal libre en sangre materna, es un método no invasivo y por lo tanto más seguro para determinar el genotipo sanguíneo fetal. En nuestro país, aún no está ampliamente disponible, sin embargo, es un avance necesario para optimizar el diagnóstico y manejo de estos embarazos. El ADN fetal libre ha sido utilizado para determinar el estado $\mathrm{RhD}$ fetal en madres $\mathrm{RhD}$ negativas con una precisión cercana al 100\% (40). En cuanto al resto de los antígenos, algunos estudios han reportado genotipificación fetal de c, E y Kell. Existe un estudio de 7 años en Holanda donde se realizó diagnóstico mediante ADN fetal libre para Sistema Rhesus D, c, E o Kell, el que se comparó con la amniocentesis o la serología neonatal. Se realizaron 362 test y se obtuvo resultados en un $97 \%$ de los casos sin ningún falso positivo o falso negativo (42).

Títulos de anticuerpos. En general, los títulos de anticuerpos no se correlacionan con la gravedad de la EHP por anticuerpos irregulares, por lo tanto, no deberían ser utilizados como base para el manejo de esta patología. Existe un estudio publicado cuyo objetivo era determinar el valor predictivo de utilizar un título crítico para asignar el riesgo de enfermedad hemolítica perinatal por los distintos anticuerpos eritrocitarios. Se estudiaron 418 casos de embarazos con anticuerpos anti eritrocitarios. Los anticuerpos presentes fueron anti- $\mathrm{D}(\mathrm{n}=359)$, anti-c $(n=34)$, anti-E $(n=19)$ y anti-K $(n=6)$. Dependiendo si los valores de los títulos eran mayores o iguales a 1:16, se establecía la severidad de la enfermedad. Los anticuerpos anti-D con un título $\geq 1: 16$ estaban presentes en el $20 \%$ de todos los casos de necesidad de transfusión. En relación a anti-c, anti-E y anti-K, solo un $4 \%$ de los casos de transfusiones tenían títulos sobre este rango. Por otro lado, los títulos $\geq 1: 16$ resultaron en ambos grupos en un $50 \%$ de los casos de fototerapia o ninguna terapia. Este trabajo permite concluir, al igual a lo observado en las distintas series de casos, que los títulos de anticuerpo no son indicadores adecuados para predecir la severidad de la enfermedad hemolítica perinatal $(43,44)$.

\section{DIAGNÓSTICO DE ANEMIA FETAL}

La espectrofotometría del líquido amniótico obtenido por amniocentesis, midiendo la diferencia en la absorción a $450 \mathrm{nM}(\Delta \mathrm{DO})$ ha sido la forma clásica de evaluar la anemia fetal en las embarazadas Rh (-) sensibilizadas. Sin embargo, este método no puede diagnosticar la anemia de causa no hemolítica, como ocurre en la supresión eritropoyética en la isoinmunización por Kell, y es un procedimiento invasivo que no está exento de riesgo, incluso muerte fetal que alcanza el $1 \%$ (45).

En el contexto de la anemia fetal (como ocurre en la EHP), se produce un aumento de la velocidad de la circulación sanguínea. Este aumento de la velocidad sanguínea se debe a la disminución de la viscosidad sanguínea y aumento del gasto cardíaco fetal secundario a la anemia. Es posible así diagnosticar la anemia fetal midiendo la velocidad del flujo sanguíneo fetal. Luego de haber evaluado distintos sitios de medida de la velocidad flujo arterial mediante Doppler, Mari y su grupo reportaron que el peak de velocidad sistólica de la arteria cerebral media (ACM) es el mejor parámetro ultrasonográfico para el diagnóstico no invasivo de anemia fetal de diferentes causas (46). Los valores del peak de la velocidad sistólica de la ACM se correlacionan muy bien con la concentración de hemoglobina fetal $(45,46)$.

Esto fue confirmado el año 2000 en un gran estudio multicéntrico que reportó una sensibilidad de $100 \%$ para la detección de anemia moderada a severa mediante la medición del peak de la velocidad sistólica de la ACM mediante Doppler (47). En relación a la anemia secundaria a isoinmunización por 
Kell, los valores de peak sistólico de ACM muestran una correlación adecuada con los valores de hemoglobina fetal, siendo por lo tanto la herramienta de elección para la evaluación de este grupo de pacientes $(48,49)$.

Las mediciones del peak sistólico de la ACM pueden iniciarse tan pronto como a las $16-18 \mathrm{se}-$ manas de embarazo y deben repetirse cada 1 a 2 semanas. Dado que valores del peak sistólico de la ACM aumentan con la edad gestacional, deben utilizarse tablas ajustadas, definiendo anemia como un peak sistólico mayor a 1,5 MoM (50).

\section{CONCLUSIÓN}

La isoinmunización feto-materna puede provocar enfermedad EHP. La principal causa sigue siendo la incompatibilidad $A B O$, seguida de la isoinmunización por RhD. Sin embargo, existen múltiples antígenos del glóbulo rojo fetal que son capaces de producir EHP en todo su espectro, siendo severa especialmente en los grupos Kell y Rhc. Existe un comportamiento diferente de la EHP en el grupo de pacientes con isoinmunización por grupos no clásicos, respecto de RhD, destacando la poca correlación entre los títulos de anticuerpo y la magnitud de la enfermedad. Además, la anemia secundaria a la isoinmunización por Kell se produce por supresión de la eritroblastosis fetal y no por hemólisis, de modo que la anemia no es diagnosticable con espectrofotometría de líquido amniótico, sino que mediante velocimetría Doppler de la ACM. Sugerimos un algoritmo de manejo basado en el screening de todas las pacientes en el control prenatal, solicitando grupo sanguíneo, Rh y test de Coombs indirecto. Si el test de coombs indirecto resulta positivo en una paciente $\mathrm{RhD}(+)$, se realiza identificación y titulación de anticuerpos irregulares y se deriva a la unidad de alto riesgo obstétrico si el anticuerpo es capaz de producir EHP. En estos casos, recomendamos el seguimiento de las pacientes sin títulos de anticuerpos, solo con control del peak de velocidad sistólica de ACM cada 2 semanas a partir de las 16 a 18 semanas y manejo según la presencia de anemia fetal (Figura 1).

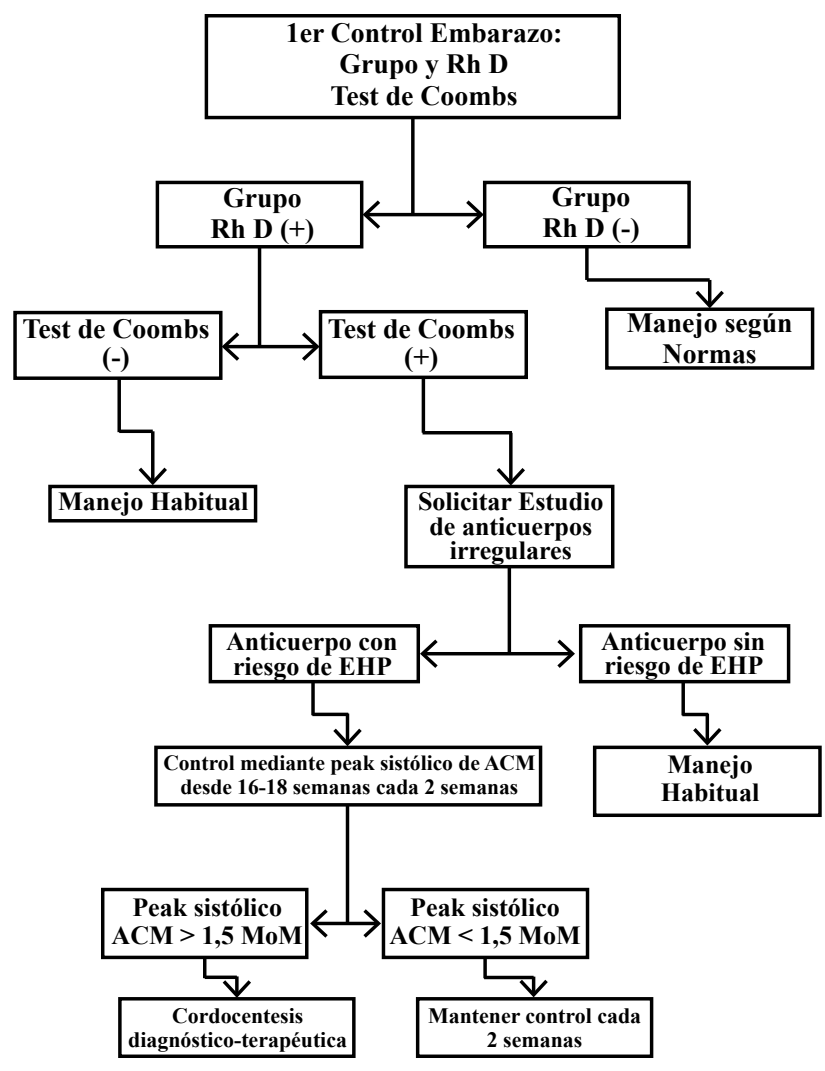

Figura 1. Esquema de manejo sugerido para detectar y tratar la isoinmunización feto-materna por anticuerpos irregulares. 


\section{REFERENCIAS}

1. Carbonne B, Castaigne V, Cynober E, Levy R, Cortey $A$, Mailloux $A$, et al. Follow-up of pregnancies with red-cell allo-immunisation: State-of-the art. Gynecol Obstet Fertil 2010;38(3):205-13.

2. Bricca P, Guinchard E, Guitton Bliem C. Management of feto-maternal red cell allo-immunizations. Transfus Clin Biol 2011;18(2):269-76.

3. Insunza A, Behnke E, Carrillo J. Enfermedad hemolítica perinatal: Manejo de la embarazada rhd negativo. Rev Chil Obstet Ginecol 2011;76(3):188-206.

4. Moise KJ. Management of rhesus alloimmunization in pregnancy. Obstet Gynecol 2008;112(1):164-76.

5. ACOG Practice Bulletin No. 75: Management of alloimmunization during pregnancy. Obstet Gynecol 2006;108(2):457-64.

6. Storry JR, Olsson ML. Genetic basis of blood group diversity. Br J Haematol 2004;126(6):759-71

7. Geifman-Holtzman O, Wojtowycz M, Kosmas E, Artal R. Female alloimmunization with antibodies known to cause hemolytic disease. Obstet Gynecol 1997;89(2):272-5.

8. Koelewijn JM, Vrijkotte TGM, Van der Schoot CE, Bonsel GJ, De Haas M. Effect of screening for red cell antibodies, other than anti-d, to detect hemolytic disease of the fetus and newborn: A population study in the Netherlands. Transfusion 2008;48(5):941-52.

9. Koelewijn JM, Vrijkotte TG, de Haas M, van der Schoot CE, Bonsel GJ. Risk factors for the presence of non-rhesus $D$ red blood cell antibodies in pregnancy. Br J Obstet Gynaecol 2009;116(5):655-64.

10. Race RR. The RhD genotype and Fisher's theory. Blood 1948;3:27-42.

11. Kozlowski CL, Lee D, Shwe KH, Love EM. Quantification of anti-c in haemolytic disease of the newborn. Transfus Med 1995;5(1):37-42.

12. Bowell PJ, Brown SE, Dike AE, Inskip MJ. The significance of anti-c alloimmunization in pregnancy. $\mathrm{Br} \mathrm{J}$ Obstet Gynaecol 1986;93:1044-8.

13. Wenk RE, Goldstein P, Felix JK. Alloimmunization by $h r^{\prime}(c)$, hemolytic disease of newborns, and perinatal management. Obstet Gynecol 1986;67:623-6.

14. Astrup J, Kornstad L. Presence of anti-c in the serum of 42 women giving birth to c positive babies: Serological and clinical findings. Acta Obstet Gynecol Scand 1977;56(3):185-8.

15. Hackney DN, Knudtson EJ, Rossi KQ, Krugh D, O'Shaughnessy RW. Management of pregnancies complicated by anti-c isoimmunization. Obstet Gynecol 2004;103(1):24-30.

16. Bowman JM, Pollock JM, Manning FA, Harman CR. Severe anti-C hemolytic disease of the newborn. Am J Obstet Gynecol 1992;166(4):1239-43.

17. Bowell PJ, Inskip MJ, Jones MN. The significance of anti-C sensitization in pregnancy. Clin Lab Haematol 1998;10(3):251-5.

18. Moran $P$, Robson SC, Reid MM. Anti-E in pregnancy. Br J Obstet Gynaecol 2000;107(11):1436-8.

19. Vaughan JI, Warwick R, Letsky E, Nicolini U, Rodeck $\mathrm{CH}$, Fisk NM. Erythropoietic suppression in fetal anemia because of kell alloimmunization. Am J Obstet Gynecol 1994;171(1):247-52.

20. Weiner CP, Widness JA. Decreased fetal erythropoiesis and hemolysis in kell hemolytic anemia. Am J Obstet Gynecol 1996;174(2):547-51.

21. Vaughan JI, Manning M, Warwick RM, Letsky EA, Murray NA, Roberts IA. Inhibition of erythroid progenitor cells by anti-kell antibodies in fetal alloimmune anemia. N Engl J Med 1998;338(12):798-803.

22. Caine ME, Mueller-Heubach E. Kell sensitization in pregnancy. Am J Obstet Gynecol 1986;154(1):85-90.

23. Mayne KM, Bowell PJ, Pratt GA. The significance of anti-kell sensitization in pregnancy. Clin Lab Haematol 1990;12(4):379-85.

24. Leggat HM, Gibson JM, Barron SL, Reid MM. Anti-Kell in pregnancy. Br J Obstet Gynaecol 1991;98(2):162-5.

25. Bowman JM, Pollock JM, Manning FA, Harman CR, Menticoglou S. Maternal kell blood group alloimmunization. Obstet Gynecol 1992;79(2):239-44.

26. McKenna DS, Nagaraja HN, O'Shaughnessy R. Management of pregnancies complicated by anti-kell isoimmunization. Obstet Gynecol 1999;93(5 Pt 1):66773.

27. Grant SR, Kilby MD, Meer L, Weaver JB, Gabra GS, Whittle MJ. The outcome of pregnancy in Kell alloimmunization. Br J Obstet Gynaecol 2000;107(4):481-5

28. Moncharmont $P$, Juron-Dupraz $F$, Doillon $M$, Vignal $M$, Debeaux P. A case of hemolytic disease of the newborn infant due to anti-K (Cellano). Acta Haematol 1991;85(1):45-6.

29. Bowman JM, Harman FA, Manning CR, Pollock JM. Erythroblastosis fetalis produced by anti-k. Vox Sang 1989;56(3):187-9.

30. Rigal D, Juron-Dupraz F, Biggio B, Jouvenceaux A. Fetal death and benign hemolytic disease of the newborn from anti-cellano alloimmunization: 2 new case reports. Rev Fr Transfus Immunohematol 1982;25(1):101-4.

31. Wikman A, Edner A, Gryfelt G, Jonsson B, Henter Jl. Fetal hemolytic anemia and intrauterine death caused by anti-m immunization. Transfusion 2007;47(5):9117.

32. De Young-Owens A, Kennedy M, Rose RL, Boyle J, O'Shaughnessy R. Anti-M isoimmunization: Management and outcome at the Ohio State University from 1969 to 1995. Obstet Gynecol 1997;90(6):962-6.

33. Kanra T, Yüce K, Ozcebe IU. Hydrops fetalis and intrauterine deaths due to anti-m. Acta Obstet Gynecol Scand 1996;75(4):415-7.

34. Davie MJ, Smith DS, White UM, Dyball D. An example of anti-s causing mild haemolytic disease of the newborn. J Clin Pathol 1972;25(9):772-3.

35. Levine P, Ferraro LR, Koch E. Hemolytic disease of the newborn due to anti-s; a case report with a review of 12 anti-s sera cited in the literature. Blood 1952;7(10):1030-7.

36. Smith G, Knott P, Rissik J, de la Fuente J, Win N. Anti$U$ and haemolytic disease of the fetus and newborn. Br J Obstet Gynaecol 1998;105(12):1318-21.

37. Dufour P, Vinatier D, Bernardi C, Ezzedine M, Fonteyne G, Monnier JC, Mannessier L. Severe fetomaternal 
anti-duffy allo-immunization. J Gynecol Obstet Biol Reprod 1991;20(6):809-14.

38. Hughes L, Rossi K, Krugh D, O'Shaughnessy R. Management of pregnancies complicated by anti-Fy(a) alloimmunization. Am J Obstet Gynecol 2004;191(6):S164.

39. Kim WD, Lee YH. A fatal case of severe hemolytic disease of newborn associated with anti-Jk(b). J Korean Med Sci 2006;21(1):151-4.

40. Spong CY, Porter AE, Queenan JT. Management of isoimmunization in the presence of multiple maternal antibodies. Am J Obstet Gynecol 2001;185(2):481-4

41. Finning K, Martin P, Summers J, Daniels G. Fetal genotyping for the $\mathrm{K}$ (Kell) and $\mathrm{rh} \mathrm{C}$, c, and $\mathrm{E}$ blood groups on cell-free fetal DNA in maternal plasma. Transfusion 2007;47(11):2126-33.

42. Scheffer PG, Van Der Schoot CE, Page-Christiaens $G$, de Haas M. Noninvasive fetal blood group genotyping of rhesus $\mathrm{D}, \mathrm{C}, \mathrm{E}$ and of $\mathrm{K}$ in alloimmunized pregnant women: Evaluation of a 7-year clinical experience. Br J Obstet Gynaecol 2011;118(11):1340-8.

43. van Dijk BA, Dooren MC, Overbeeke MA. Red cell antibodies in pregnancy: There is no 'critical titre'. Transfus Med 1995;5(3):199-202.

44. van Wamelen DJ, Klumper FJ, de Haas M, Meerman $\mathrm{RH}$, van Kamp IL, Oepkes D. Obstetric history and antibody titer in estimating severity of Kell alloimmunization in pregnancy. Obstet Gynecol 2007;109(5):1093-8.
45. Vargas D, Carvajal J. El Doppler de la arteria cerebral media reemplaza a la espectrofotometría del líquido amniótico como el estándar en el manejo de la isoinmunización fetal. Rev Chil Obstet Ginecol 2007;72(4):229-35.

46. Mari G, Adrignolo A, Abuhamad AZ, Pirhonen J, Jones DC, Ludomirsky A, Copel JA. Diagnosis of fetal anemia with doppler ultrasound in the pregnancy complicated by maternal blood group immunization. Ultrasound Obstet Gynecol 1995;5(6):400-5.

47. Mari G, Deter RL, Carpenter RL, Rahman F, Zimmerman R, Moise Jr KJ. Noninvasive diagnosis by doppler ultrasonography of fetal anemia due to maternal redcell alloimmunization. N Engl J Med 2000;342(1):914.

48. Rimon E, Peltz R, Gamzu R, Yagel S, Feldman B, Chayen B. Management of Kell isoimmunization-evaluation of a doppler-guided approach. Ultrasound Obstet Gynecol 2006;28(6):814-20.

49. van Dongen $H$, Klumper FJ, Sikkel E, Vandenbussche FP, Oepkes D. Non-invasive tests to predict fetal anemia in kell-alloimmunized pregnancies. Ultrasound Obstet Gynecol 2005;25(4):341-5.

50. Moise KJ, Argoti PS. Management and prevention of red cell alloimmunization in pregnancy: A systematic review. Obstet Gynecol 2012;120(5):1132-9. 